\title{
Colored flux tube in the Euclidean spacetime
}

\author{
Vladimir Dzhunushaliev* \\ February 8, 2020 \\ Institut für Mathematik, Universität Potsdam, D-14469, Potsdam, Germany \\ and \\ Dept. Phys. and Microel. Engineer., Kyrgyz-Russian Slavic University \\ Bishkek, Kievskaya Str. 44, 720000, Kyrgyz Republic
}

\begin{abstract}
The flux tube solution in the Euclidean spacetime with the color longitudinal electric field in the $\mathrm{SU}(2)$ Yang - Mills - Higgs theory with broken gauge symmetry is found. Some arguments is given that this flux tube is a pure quantum object in the $\mathrm{SU}(3)$ quantum theory reduced to the $\mathrm{SU}(2) \mathrm{Yang}$ - Mills - Higgs theory.
\end{abstract}

\section{Introduction}

The confinement problem in quantum chromodynamics (QCD) is naturally connected with the existence of a hypothesized flux tube filled with a color longitudinal electric field and stretched between quark and antiquark. Such tube confines quark and antiquark in a pair and does not give any possibility to destroy such pair. Evidently the derivation of the colored non-Abelian flux tube is impossible in classical gauge field theory ${ }^{1}$ and still remains an unresolved problem in QCD. In this notice we would like to show that the flux tube solution exists in the SU(2) Yang - Mills - Higgs theory with broken gauge symmetry. The gauge symmetry breakdown is a quantum phenomenon therefore we are searching the flux tube solution in the classical theory with some quantum additional term. It means that we consider an approximate model of the flux tube with the color electric field. At the end of this notice we will argue that the above mentioned Yang - Mills - Higgs theory can be obtained as an approximation of quantum SU(3) gauge theory where the $\mathrm{SU}(3)$ gauge potential is splitted on two parts: the first one is the $\mathrm{SU}(2)$ gauge potential $(\mathrm{SU}(2)$ is a small subgroup of $\mathrm{SU}(3): S U(2) \in S U(3))$ and the second one is the coset $S U(3) / S U(2)$. The $\mathrm{SU}(2)$ degrees of freedom remain almost classical but the coset degrees of freedom are completely quantum and can be presented as a scalar field (physically it is a condensate of the coset fields). In this presentation $\mathrm{SU}(2)$ and coset fields are similar to a magnetic field and wave function of Cooper pairs in a superconductor.

\footnotetext{
*E-mail: dzhun@hotmail.kg

${ }^{1}$ altough in dual theories exist the well known Nilesen-Olesen flux tube solutions 1 filled with a longitudinal Abelian magnetic field $H_{z}$.
} 


\section{Initial equations}

Let us start from the Yang - Mills - Higgs field equations in the Euclidean spacetime with broken gauge symmetry

$$
\begin{aligned}
\mathcal{D}_{\nu} F^{a \mu \nu} & =g \epsilon^{a b c} \phi^{b} \mathcal{D}^{\mu} \phi^{c}-m^{2} A^{a \mu} \\
\mathcal{D}_{\mu} \mathcal{D}^{\mu} \phi^{a} & =-\lambda \phi^{a}\left(\phi^{b} \phi^{b}-\phi_{\infty}^{2}\right)
\end{aligned}
$$

here $F_{a \mu \nu}=\partial_{\mu} A_{\nu}^{a}-\partial_{\nu} A_{\mu}^{a}+g \epsilon^{a b c} A_{\mu}^{b} A_{\nu}^{c}$ is the field tensor for the $\mathrm{SU}(2)$ gauge potential $A_{\mu}^{a} ; a, b, c=1,2,3$ are the color indices; $D_{\nu}[\cdots]^{a}=\partial_{\nu}[\cdots]^{a}+g \epsilon^{a b c} A_{\mu}^{b}[\cdots]^{c}$ is the gauge derivative; $\phi^{a}$ is the Higgs field; $g$ and $\phi_{\infty}^{a}$ are some constants; $m^{2} A_{\mu}^{a}$ is the most important term for us which destroys the gauge invariance of the Yang - Mills - Higgs theory.

The solution we search in the following form

$$
A_{\tau}^{1}=\frac{f(\rho)}{g} ; \quad A_{z}^{2}=\frac{v(\rho)}{g} ; \quad \phi^{3}=\frac{\phi(\rho)}{g}
$$

here $z, \rho, \varphi$ are cylindrical coordinate system, $\tau$ is the Euclidean time. The substitution to the Euclidean Yang - Mills - Higgs equations gives us

$$
\begin{aligned}
f^{\prime \prime}+\frac{f^{\prime}}{\rho} & =f\left(\phi^{2}+v^{2}-m^{2}\right) \\
v^{\prime \prime}+\frac{v^{\prime}}{\rho} & =v\left(\phi^{2}+f^{2}-m^{2}\right) \\
\phi^{\prime \prime}+\frac{\phi^{\prime}}{\rho} & =\phi\left[f^{2}+v^{2}+\lambda\left(\phi^{2}-\phi_{\infty}^{2}\right)\right]
\end{aligned}
$$

here we redefined $g \phi \rightarrow \phi, g f \rightarrow f, g v \rightarrow v$ and $\lambda / g^{2} \rightarrow \lambda$. The similar equations in the Lorentzian spacetime with the presence of $A_{\varphi}^{a}$ (and consequantley with a magnetic field $H_{z}^{a}$ ) was investigated in Ref. [2] and it was shown that exist: (1) the flux tube filled with electric/magnetic fields on the background of an external constant magnetic/electric field; (2) the Nielsen-Olesen flux tube dressed with transversal color electric and magnetic fields.

We will consider the simplest case $f=v$. Then we have

$$
\begin{aligned}
& f^{\prime \prime}+\frac{f^{\prime}}{x}=f\left(\phi^{2}+f^{2}-m^{2}\right), \\
& \phi^{\prime \prime}+\frac{\phi^{\prime}}{x}=\phi\left[2 f^{2}+\lambda\left(\phi^{2}-\phi_{\infty}^{2}\right)\right]
\end{aligned}
$$

here we redefined $f / \alpha \rightarrow f, \phi / \alpha \rightarrow \phi$ and $m / \alpha \rightarrow m, \phi_{\infty} / \alpha \rightarrow \phi_{\infty}$ and $\rho \alpha \rightarrow x ; \alpha$ is some constant which will be defined later.

\section{Numerical investigation}

We will solve the equations set (7) (8) with an iterative procedure. On the $i$ step Eq. (7) has the following form

$$
f_{i}^{\prime \prime}+\frac{f_{i}^{\prime}}{x}=f_{i}\left(\phi_{i-1}^{2}+f_{i}^{2}-m_{i}^{2}\right)
$$


here $\phi_{i-1}$ was defined on $(i-1)$ step and the null approximation is $\phi_{0}=2-1 / \cosh ^{2}(x / 4)$. The numerical investigation for Eq. (9) shows that there is a number $m_{i}^{*}$ for which: for $m_{i}>m_{i}^{*}$ the solution is singular $f_{i}(x) \rightarrow-\infty$ by $x \rightarrow x_{0}$ and for $m_{i}<m_{i}^{*}$ the solution is also singular $f_{i}(x) \rightarrow+\infty$ by $x \rightarrow x_{0}$. It means that for the value $m_{i}=m_{i}^{*}$ the solution $f(x)$ is regular one. The value $m_{i}^{*}$ can be defined with the method of iterative approximation.

The next step on the $i$ iteration is solving of Eq. (8)

$$
\phi_{i}^{\prime \prime}+\frac{\phi_{i}^{\prime}}{x}=\phi_{i}\left[2 f_{i}^{2}++\lambda\left(\phi_{i}^{2}-\phi_{i \infty}^{2}\right)\right] .
$$

The numerical investigation for this equation shows that there is a number $\phi_{i \infty}$ for which: for $\phi_{i \infty}>\phi_{i \infty}^{*}$ the function $\phi_{i}(x)$ oscillates with decreasing amplitude, for $\phi_{i \infty}<\phi_{i \infty}^{*}$ the function $\phi_{i}(x) \rightarrow+\infty$ by $x \rightarrow x_{0}$. The number $\phi_{i}^{*}$ defines a regular solution $\phi_{i}(x)$.

The value $f(0)$ can be arbitrary but we choose them by such a way that $f(0) / \alpha=0.5$. Thus in the equations set (7) (8) we have only two independent parameters $\lambda$ and $\phi(0)$. In the calculations presented here we take $\lambda=0.1$ and $\phi(0) / \alpha=1.0$.

The iterative process described above gives us the $m_{i}^{*}$ and $\phi_{i}^{*}$ presented on Table 1 The functions

\begin{tabular}{|c|c|c|c|c|}
\hline $\mathrm{i}$ & 1 & 2 & 3 & 4 \\
\hline$m_{i}^{*}$ & $1.342794 \ldots$ & $1.362562 \ldots$ & $1.359847 \ldots$ & $1.359851 \ldots$ \\
\hline$\phi_{i}^{*}$ & $1.613484 \ldots$ & $1.601260 \ldots$ & $1.606675 \ldots$ & $1.606186 \ldots$ \\
\hline
\end{tabular}

Table 1: The iterative parameters $m_{i}^{*}$ and $\phi_{i}^{*}$.

$f_{i}(x)$ and $\phi_{i}(x)$ for $i=1,2$ are presented on Fig's 10 2

The electric and magnetic fields are

$$
\begin{aligned}
E_{z}^{3}(x) & =f(x) v(x)=f^{2}(x), \\
-E_{\rho}^{1}(x) & =f^{\prime}(x), \\
H_{\varphi}^{2}(x) & =x \epsilon_{\varphi \rho z} F^{\rho z}=x f^{\prime}(x)
\end{aligned}
$$

These fields are presented on Fig. 3

It is easy to show that the asymptotical behaviour of the regular solutions $f^{*}(x)$ and $\phi^{*}(x)$ is

$$
\begin{aligned}
& f^{*}(x)=f_{0} \frac{e^{-x \sqrt{\phi_{\infty}^{* 2}-m^{* 2}}}}{\sqrt{x}}+\cdots, \\
& \phi^{*}(x)=\phi_{\infty}^{*}-\phi_{0} \frac{e^{-x \sqrt{2 \lambda \phi_{\infty}^{* 2}}}}{\sqrt{x}}+\cdots .
\end{aligned}
$$

\section{Quantum interpretation of the obtained colored flux tube}

In Ref. [3] it is shown that having some assumptions about the non-perturbative 2 and 4-points Greens functions in the SU(3) quantum Yang - Mills theory it can be reduced to the classical SU(2) Yang - Mills - Higgs theory plus some extra term which is zero for the ansatz (3). In shorten this $S U(3) \rightarrow S U(2)$ reduction can be presented by the following manner (here we follow to Ref. [3]). At first we decompose the $\mathrm{SU}(3)$ gauge potential on ordered and disordered phases: 


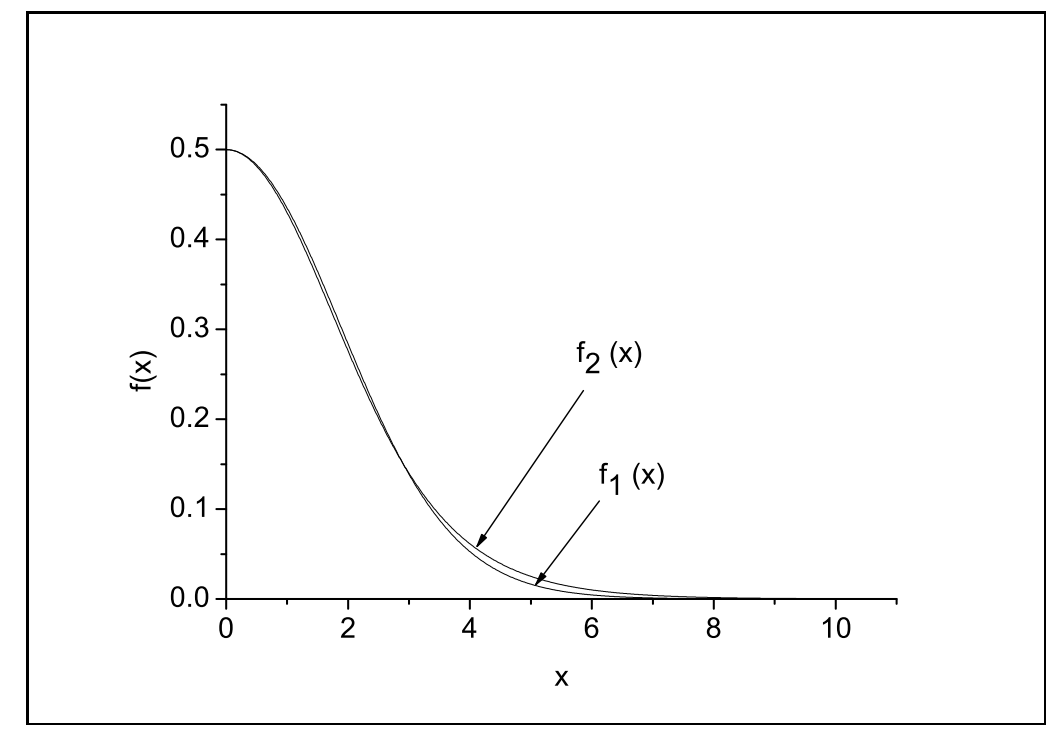

Figure 1: The functions $f_{1}(x)$ and $f_{2}(x)$. The first and second iterations practically coincide.

1. The gauge field components $A_{\mu}^{a} \in S U(2), a=1,2,3$ belonging to the small subgroup $S U(2) \subset S U(3)$ are in an ordered phase. It means that

$$
\left\langle A_{\mu}^{a}(x)\right\rangle \approx\left(A_{\mu}^{a}(x)\right)_{c l} .
$$

The subscript means that this is the classical field. It is assumed that in the first approximation these degrees of freedom are classical and is described by $\mathrm{SU}(2)$ Yang - Mills equations. $\langle\ldots\rangle$ is a quantum average.

2. The gauge field components $A_{\mu}^{m}(\mathrm{~m}=4,5, \ldots, 8)$ and $\left.A_{\mu}^{m} \in S U(3) / S U(2)\right)$ belonging to the coset $\mathrm{SU}(3) / \mathrm{SU}(2)$ are in a disordered phase (or in other words it is a condensate). It means that

$$
\left\langle A_{\mu}^{m}(x)\right\rangle=0, \text { but }\left\langle A_{\mu}^{m}(x) A_{\nu}^{n}(x)\right\rangle \neq 0 .
$$

These degrees of freedom are pure quantum degrees and are involved in the equations for the ordered phase as an averaged field distribution of coset components.

In Ref. 3] was made the following assumptions and simplifications:

1. The correlation between coset components $A_{\mu}^{m}(y)$ and $A_{\nu}^{n}(x)$ in two points $x^{\mu}$ and $y^{\mu}$ is

$$
\left\langle A_{\mu}^{m}(y) A_{\nu}^{n}(x)\right\rangle=-\eta_{\mu \nu} \mathcal{G}^{m n}(y, x) .
$$

The function $\mathcal{G}^{m n}(y, x)$ can be presented in one function approximation as

$$
\mathcal{G}^{m n}(y, x)=-\frac{1}{3} f^{m p b} f^{n p c} \phi^{b}(y) \phi^{c}(x)
$$

where $f^{a b c}$ is the structural constants of the $\mathrm{SU}(3)$ group. 


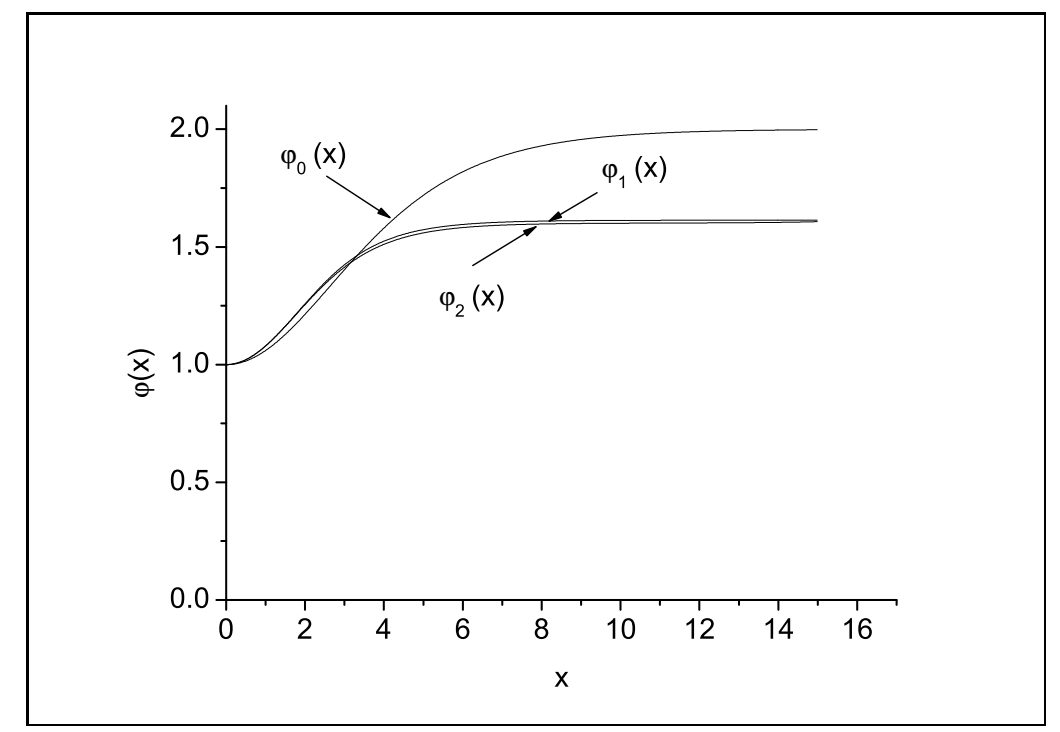

Figure 2: The functions $\phi_{0}(x), \phi_{1}(x)$ and $\phi_{2}(x)$. The first and second iterations practically coincide.

2. There is not correlation between ordered (classical) and disordered (quantum) phases

$$
\left\langle f\left(a_{\mu}^{a}\right) g\left(A_{\nu}^{m}\right)\right\rangle=f\left(a_{\mu}^{a}\right)\left\langle g\left(A_{\mu}^{m}\right)\right\rangle
$$

where $f$ and $g$ are arbitrary functions.

3. In the first approximation the 4-point Green's function is

$$
\begin{array}{r}
\left\langle A_{\alpha}^{m}(x) A_{\beta}^{n}(y) A_{\mu}^{p}(z) A_{\nu}^{q}(u)\right\rangle= \\
\left(E_{1, a b c d}^{m n p q} \eta_{\alpha \beta} \eta_{\mu \nu}+E_{2, a b c d}^{m p n q} \eta_{\alpha \mu} \eta_{\beta \nu}+E_{3, a b c d}^{m q n p} \eta_{\alpha \nu} \eta_{\beta \mu}\right) \phi^{a}(x) \phi^{b}(y) \phi^{c}(z) \phi^{d}(u)
\end{array}
$$

here $E_{1, a b c d}^{m n n q}, E_{2, a b c d}^{m p n q}, E_{3, a b c d}^{m q n p}$ are some constants. The main idea proposed in Ref. [3] is that the initial $\mathrm{SU}(3)$ Lagrangian

$$
\mathcal{L}_{S U(3)}=-\frac{1}{4} F_{\mu \nu}^{A} F^{A \mu \nu}, A=1,2, \cdots 8 .
$$

after above mentioned assumptions and simplifications can be reduced to the SU(2) Yang - Mills - Higgs Lagrangian

$$
\mathcal{L}_{S U(2)}=-\frac{1}{4} F_{\mu \nu}^{a} F^{a \mu \nu}+\frac{1}{2}\left(\partial_{\mu} \phi^{a}-\frac{g}{2} \epsilon^{a b c} A_{\mu}^{b} \phi^{c}\right)^{2}+\frac{m_{\phi}^{2}}{2}\left(\phi^{a} \phi^{a}\right)-\lambda\left(\phi^{a} \phi^{a}\right)^{2}+\frac{g^{2}}{2} a_{\mu}^{b} \phi^{b} a^{c \mu} \phi^{c} .
$$

Here is also assumed that there is the gauge symmetry breakdown lieading to the term $\frac{m_{\phi}^{2}}{2}\left(\phi^{a} \phi^{a}\right)$. The first term $F_{\mu \nu}^{a} F^{a \mu \nu}$ is the Lagrangian for the ordered phase $A_{\mu}^{a}$ (the $\mathrm{SU}(2)$ Lagrangian) and the next 3 terms are the Lagrangian for the disordered (condensate) phase (Higgs Lagrangian). There is an 


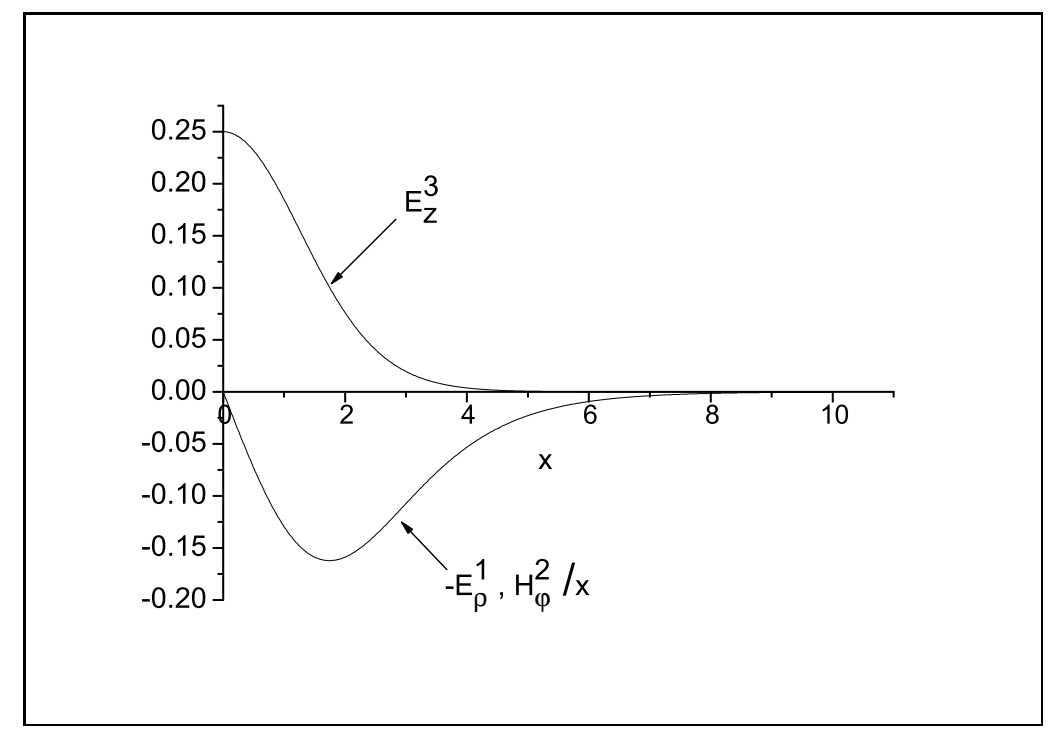

Figure 3: The electric fields $E_{z}^{3}(x), E_{\rho}^{1}(x)$ and magnetic field $H_{\varphi}^{2}(x)=-E_{\rho}^{1}(x)$

additional gauge noninvariant term $\frac{g^{2}}{2} a_{\mu}^{b} \phi^{b} a^{c \mu} \phi^{c}$. For the ansätz (3) the corresponding terms in field equations are zero and it is nonimportant for us.

Finally, in the context of offered here $S U(3) \rightarrow S U(2)$ reduction the colored flux tube obtained above is a pure quantum object in the $S U(3)$ Yang - Mills theory.

\section{Conclusions}

In this notice we have shown that in the SU(2) Yang - Mills - Higgs theory can exist the flux tube solutions filled with the color longitudinal electric field for which it is necessary to have a gauge symmetry breakdown. The origin of such breaking can be a quantum mechanism similar to a Coleman - Weinberg mechanism [4] in $\lambda \phi^{4}$-theory. The essential difference is that in our case the symmetry breakdown mechanism should be non-perturbative one and its derivation now is the very big problem because we have not any analytical non-perturbative technique for the quantum field theories with strong interactions.

It is interesting to note that the regular solution for the colored flux tube exists only for a discrete spectrum of $m^{*}$ and $\phi^{*}$ parameters. Probably it is an indication of quantum origin of this object like to a discrete spectrum of energy levels of a quantum particle in a potential well.

One can underline the difference between the Nielsen - Olesen and colored flux tubes: the first one is a topological solution but the another one is a pure dynamical object without any topological properties.

Finally, the main result of this notice is that the confinement problem is closely connected with the problem of the symmetry breakdown: if symmetry breaking takes place then exists a colored flux tube solution. The mathematical problems here is the absense of any analytical technique for non-perturbative calculations in gauge field theories. It is possible that such technique can be based on the Heisenberg idea about the quantization of a non-linear spinor field [5]. 


\section{Acknowledgments}

I am very grateful to the Alexander von Humboldt foundation for the financial support of this work and H.-J- Schmidt for the invitation to research in Potsdam University.

\section{References}

[1] H.B. Nielsen and P. Olesen, Nucl. Phys. B61, 45 (1973).

[2] V. Dzhunushaliev, "Flux tube dressed with color electric $E_{\rho, \phi}^{a}$ and magnetic $H_{\rho, \phi}^{a}$ fields" hep-ph/0306203 V. Dzhunushaliev, "Electric/magnetic flux tube on the background of magnetic/electric field ", hep-th/0302215

[3] V. Dzhunushaliev and D. Singleton, Mod. Phys. Lett., A18, 955(2003); V. Dzhunushaliev and D. Singleton, "Effective 't Hooft-Polyakov monopoles from pure SU(3) gauge theory", hep-ph/0306202

[4] S. Coleman and E. Weinberg, Phys. Rev. D7, 1888 (1973).

[5] W. Heisenberg, Introduction to the unified field theory of elementary particles., Max - Planck - Institut für Physik und Astrophysik, Interscience Publishers London, New York, Sydney, 1966; W. Heisenberg, Nachr. Akad. Wiss. Göttingen, N8, 111(1953); W. Heisenberg, Zs. Naturforsch., 9a, 292(1954); W. Heisenberg, F. Kortel und H. Mütter, Zs. Naturforsch., 10a, 425(1955); W. Heisenberg, Zs. für Phys., 144, 1(1956); P. Askali and W. Heisenberg, Zs. Naturforsch., 12a, 177(1957); W. Heisenberg, Nucl. Phys., 4, 532(1957); W. Heisenberg, Rev. Mod. Phys., 29, 269(1957). 